The Digital Object Identifier - DOI: 10.37952/ROI-jbc-01/20-61-1-14

Submitted on January 24, 2020.

\title{
On un-harmonic vibrations of diatomic molecule and atomic dimer
}

\author{
(C) Ikhtier H. Umirzakov, ${ }^{+}{ }^{+}$and Edward N. Mayer \\ Novosibirsk State University. Pirogova St., 2. Novosibirsk, 630090. Russia. \\ Phone: +7 (383) 354-20-17. E-mail: tepliza@academ.org
}

*Supervising author; ${ }^{+}$Corresponding author

Keywords: un-harmonic corrections, vibrations, diatomic molecule, dimer, potential.

\begin{abstract}
According to modern concepts, the interaction potential as a function of the interatomic distance has a single minimum, has no other extremum points, and does not have inflection points, where its derivative is zero. Usually, to take into account harmonic vibrations and the effect of anharmonic effects on these vibrations, the interaction potential is expanded in the Taylor series and a quadratic term is taken into account, which takes into account harmonic vibrations, and third and fourth order corrections for deviations of the interatomic distance from its equilibrium value, taking into account anharmonic effects. These three members of the Taylor series constitute an approximate interaction potential. To take into account the anharmonic effects in the partition function of the diatomic molecule in the Boltzmann factor, an exponential factor is selected that takes into account the anharmonic part of the above approximate potential, and this factor is expanded in the Taylor series, the integration limits are changed to plus and minus infinity, the integration is carried out and the first few members of the power series are left on return temperature. Obviously, the approximate potential, like the initial potential, should not have other extremum points, except for a single minimum, and inflection points, where its derivative is equal to zero. In this paper, it is shown that: if the coefficient in front of the fourth-order term in the approximate potential is negative, then the approximate potential has two false maxima; if it is zero, then there is one false maximum; if it is positive, then the potential can have one false minimum and one false maximum or one inflection point where the derivative of the potential is equal to zero. The conditions imposed on the interaction potential are found when the anharmonic effects in the vibrations of a diatomic molecule can be approximately taken into account by corrections of the third and fourth orders to the quadratic harmonic potential. It is shown that this approximation can be used in the case of the interaction of atoms of a diatomic molecule by means of the MieGrüneisen and Lennard-Jones potential.
\end{abstract}

\section{References}

[1] L.D. Landau, E.M. Livshits. Theoretical physics. Statistical physics. Moscow: Science. Vol.V. Iss.1. 1976. 584p. (russian)

[2] R. Feynman. Statistical mechanics. Lecture course. Moscow: Mir. 1975. 409p. (russian) 